\title{
Víctor Albis González
}

\author{
1939-2017
}

Víctor Samuel Albis González nació en Sincelejo el 14 de noviembre de 1939 en una familia de educadores formada por Víctor Hugo Albis Villalba y Julieta González-Tapia Gómez. Su abuelo materno Samuel González-Tapia tenía un colegio para varones, el González Tapia, y su madre quien era Normalista Superior de la Normal de Cartagena fundó el Instituto de Cultura Femenina para señoritas. Su padre además de comerciante era poeta. Víctor Samuel hizo sus primeros estudios en su ciudad natal, en el Colegio del Presbítero Antonio Prieto, "un español mal hablado", y luego para hacer el bachillerato fue enviado a Bogotá. Estuvo interno en el Colegio de Santo Tomás, en el Liceo de la Salle y terminó sus estudios en el Colegio del Rosario. En 1958 fue admitido a la carrera de Matemáticas de la Universidad Nacional (UN), a la que ingresara sin estudios previos de ingeniería, algo que ningún alumno había logrado anteriormente. Se graduó en 1963 con un estudio titulado Potencia exterior topológica de un modelo.

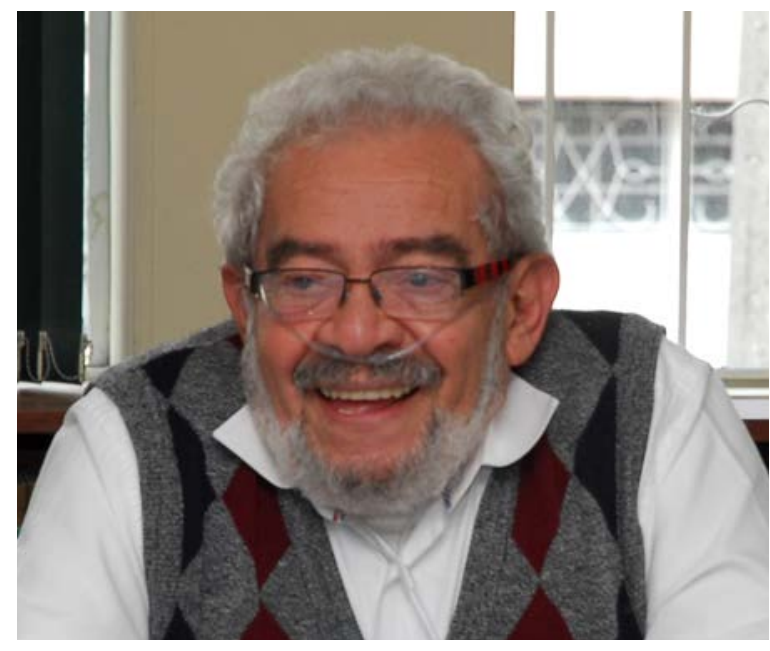

Distinguido como el mejor estudiante de su promoción, Víctor Samuel recibió una beca y viajó a estudiar en la Universidad de Paris IV. Allí permaneció por dos años, asistió a los cursos de álgebra y teoría de números dictados por Pierre Samuel y Claude Chevalley. Como la beca era precaria y había viajado con su familia, doña Regina Feliz y su hija, Rosario, la situación económica se hizo insostenible impidiéndole concentrarse en sus estudios por lo que regresó a Bogotá dos años después para retomar su cargo en el Departamento de Matemáticas de la UN en donde impartió el curso de cálculo avanzado. Posteriormente, en 1969 viajó a la Universidad de Boulder, Colorado, en los Estados Unidos, con una beca Ford y obtuvo su doctorado en matemáticas en 1972.

En 1960 aun como estudiante, Víctor revivió la Revista de Matemáticas Elementales (RME), fundada en 1952, publicación conjunta de las Universidades Nacional y de los Andes, la que en 1967 Víctor mismo convertiría en dos, a saber, la Revista Colombiana de Matemáticas (RCM) de nivel investigativo y el Boletín de Matemáticas dedicado a profesores de enseñanza media y estudiantes universitarios. Con apoyo especial del tesorero de la Sociedad Colombiana de Matemáticas (SCM), Camilo Rubiano, se le presentaron estas revistas al director del Departamento de Matemáticas, profesor Ricardo Losada, quien se sorprendió por la decisión que el “enfant terrible” había tomado sin consultarlo. No obstante, resolvió apoyarlo. Años más tarde, Víctor delegó en Hernando Pérez la dirección del Boletín mientras que el mismo retenía la correspondiente a la Revista, función que desempeñó hasta 1978.

Quien escribe quiere destacar este aspecto del profesor Albis quien, desde ese entonces, estuvo ligado de una u otra forma, a las revistas en asocio con la SCM, publicaciones que han permitido que el Departamento de Matemáticas de la UN cuente con un medio de difusión para el avance permanente del conocimiento. La calidad de la RCM está marcada por el rigor en la selección de los artículos y por su impecable presentación, la que, además, ha sido en términos matemáticos, una prueba de existencia de la comunidad matemática colombiana y un vínculo en extremo valioso para el contacto con el mundo exterior especialmente si se considera que nuestra incipiente comunidad solía hacer muy escasa presencia en eventos internacionales. La RCM, desde su antecesora la RME, permitió el canje con revistas de matemáticas de primer nivel en todo el mundo, un vínculo de valor único. Es así como por muchos años, la Biblioteca de Matemáticas y Estadística de la UN, fue la más importante de Colombia. Actualmente se la encuentra repartida entre la Biblioteca de Ciencia y Tecnología, la Hemeroteca y la Biblioteca Central. La revista ha tenido continuidad desde entonces, lo cual es una verdadera proeza en nuestro medio.

El Boletín, en cambio, ha tenido varias etapas. Fue un órgano de difusión conjunto entre el DM y la SCM, pero a finales de los años 70 bajo la presidencia de Alonso Takahashi se decide, por razones que no son del caso mencionar, fundar las Lecturas Matemáticas como órgano oficial de difusión de la SCM y dejar a cargo del DM el Boletín. Víctor Albis perteneció al Comité Editorial de Lecturas Matemáticas desde 1997 y fue su editor hasta su fallecimiento. 
La vida de Víctor Albis fue, por lo tanto, una vida dedicada a la labor de difusión de nuestros trabajos y el científico capaz de abrir la puerta a matemáticos extranjeros que ahora publican en nuestras revistas. Comencé este escrito presentando a Víctor Albis, como el editor. Editor de revistas, miembro de comités editoriales, y revisor muy riguroso de múltiples trabajos de matemáticas, riguroso tanto en lo matemático como en la calidad de la redacción y la ortografía. Pero, además de editor, su trayectoria académica tuvo otras facetas, las que se presentan a continuación.

Víctor Albis, el historiador. En la biblioteca de su abuelo, Víctor se interesó por los libros viejos de matemáticas y ciencia como los Elementos de Geometría de Legendre en la edición venezolana, El valor de la ciencia de Poincaré, Cosmos de Humboldt y la Astronomía de Flanmarión. Comenzó entonces a hacer reseñas y publicarlas en la RME. Ese interés se convirtió en el Programa de Investigaciones Históricas de la Matemática en Colombia, proyecto que fue presentado con el apoyo de la SCM a Colciencias y que fuera uno de los primeros proyectos de investigación avalados (1974) por la recién fundada Colciencias. Tengo el honor de haber sido incluida desde entonces en este proyecto histórico. El proyecto tiene como objetivo recuperar, recopilar, analizar y construir un catálogo de la producción matemática de los colombianos. Hoy este proyecto puede mostrar resultados muy valiosos como se aprecia en las páginas de la SCM y de la Academia Colombiana de Ciencias. Una base de datos con cerca de 5000 registros de la producción matemática de los colombianos, que abarca trabajos desde el siglo XIX y que está disponible en el servidor de la SCM (www.scm.org.co). Contamos, además, con la página web (www.accefyn.org. co/historia-matematica/mathematica/miscelanea.htm) en el servidor de la Academia Colombiana de Ciencias. Ésta contiene una galería de matemáticos colombianos, parte de las publicaciones que se han hecho sobre los documentos encontrados, de verdadero valor patrimonial, y una bibliografía con documentos de los siglos XIX y XX. Ello permite citar el cuaderno del primer curso de cálculo impartido en el país, en 1951con las lecciones de Aimé Bergeron, el análisis de los trabajos de Julio Garavito e Indalecio Liévano sobre el postulado de Euclides, o la carta de Hermite en la que le resuelve un sencillo problema de geometría a Luis María Lleras. Desafortunadamente el proyecto se estancó hace unos años por falta de recursos.

El trabajo realizado con Luis Ignacio Soriano (1903-1973) sobre los números inconmensurables de Liévano en su Tratado Elemental de Aritmética de 1856, le abrió las puertas de la revista Historia Mathemática, como miembro del Comité Editorial y fue además nombrado miembro de la International Comisision for the History of Mathematics. Allí se hizo conocido especialmente por sus reseñas, de las cuales se cuentan más de cien entre ésta y otras revistas.

Conocer el origen y desarrollo de una idea permite entender mejor la idea misma y deberían facilitar su enseñanza. Por ello Víctor organizó en 1974 un Seminario de Historia de las Matemáticas el cual duró varios años a su cargo. Junto con Alberto Campos abrieron el camino para que hoy en día el área de la historia y de la filosofía de la matemática empiecen a ser reconocidas en nuestro medio como áreas de estudio válidas para un matemático.

Víctor y la etno-matemática. Ese interés por nuestra historia lo llevó a otro tema de investigación: herramientas matemáticas para la antropología. Antropología y Matemáticas fue una interesante investigación que realizó con el destacado antropólogo Guillermo Páramo. Sus trabajos sobre los grupos de simetría que se encuentran en los dibujos que se hallan en los más diversos objetos precolombinos como las vasijas de cerámica, los poporos, narigueras y demás. Las matemáticas pueden facilitar la clasificación de los mismos para distinguirlos como pertenecientes a una cultura o a otra. Igualmente, un estudiante realizó como su trabajo de grado el análisis de los sistemas de numeración de la familia macrochicha, quienes fueron pioneros en nuestro país.

Víctor Albis el algebrista. El álgebra y la teoría de números fueron sus áreas de interés en la matemática "pura". Los resultados de sus investigaciones Anillos euclídeos con Raj Markanda de la Universidad del Valle, Teoría aritmética de polinomios y funciones algebraicas, Funciones zeta locales de Igusa. Racionalidad en característica positiva para ciertos tipos de polinomios con Wilson Zúñiga, reflejados todos ellos en sus publicaciones y en la formación de aquellos estudiantes a quienes dirigió sus trabajos de grado o sus tesis en los diferentes niveles de formación académica. Su producción puede apreciarse, justamente, en la base de datos que hemos construido y que puede ser consultada en la red.

Víctor el docente. Víctor fue un profesor “cuchilla” como decimos en nuestro argot regional. Creo que esa dureza es pecado de juventud, pecado estoy segura que, en mayor o menor medida, hemos cometido los que hemos ido aprendiendo el oficio de la docencia. Víctor - como la gran mayoría de veteranos en el oficio - se volvió un profesor madre, al que se acercan muchos estudiantes para escuchar sus consejos, para que sea su guía y ello no solo desde el punto de vista académico. Él se consideraba un chozno de Hilbert, y puedo afirmar que la descendencia por su lado continúa. En esta faceta hay que destacar que siempre entregó a sus alumnos sus propias notas. Nos legó dos libros pioneros: Cálculo I (1969) escrito con Yu Takeuchi y Temas de Aritmética y Álgebra (1976).

Víctor el administrador. Como primer Rector de la Universidad de Sucre realizó una gran labor en pro de la educación superior en su Departamento. Ello le valió varias distinciones como la de "Mejor Ejecutivo del Año "otorgada por Cámara Júnior de Colombia, capítulo de Sucre, en 1981; la “Medalla Alfredo González Rubio” de la Cámara Júnior de Colombia en 
1982; y la Orden “Mariscal Sucre” del Gobierno de Departamental de Sucre por servicios distinguidos en 1984. En la UN ocupó varios cargos: Jefe de Planeación, Director del Departamento de Bibliotecas, Director del Posgrado de Matemáticas. La Universidad ha reconocido su aporte integral a la Universidad con las distinciones de "Maestro Universitario" en 1986, la Medalla al “Mérito Universitario” en 1995, y en 2004 le fue otorgada la Orden “Gerardo Molina”, la más alta distinción que otorga la UN a sus profesores activos. Fue Presidente de la SCM entre 1990 y 1993 y, como tal, apoyó la organización del “IV Coloquio Internacional de Historia y Filosofía de las Matemáticas y Primero en Educación Matemática” en Colombia realizado en 1993 con una gran acogida y más de 300 participantes. Fue Secretario de la Academia Colombiana de Ciencias Exactas, Físicas y Naturales (1990-1992), Director de su biblioteca (1994-2002), y Director de la Gaceta.

Víctor el esposo y padre. Regina Feliz, su esposa desde 1963, brindó su decidido apoyo para que Víctor pudiera realizar las numerosas tareas que, por la matemática, y por la educación en este país, se empeñó en su larga y meritoria vida académica. Tres hijos, Rosario, Samuel y María Alejandra y seis nietos, Nicolás, Alejandro, Sarita, Daniel, Lucca y Grace Marie, fueron su alegría, soporte afectivo, su orgullo, y, sin duda, motor para su vida. A todos ellos van nuestro más profundo sentimiento de condolencia. Paz en su tumba...

Clara Helena Sánchez B.

Departamento de Matemáticas

Universidad Nacional de Colombia - Bogotá.

Esta nota sobre la vida y obra de Víctor Albis está basada en la presentación que de él hiciera cuando recibió el Premio Nacional de Matemáticas en 2007. Trabajo publicado en Lecturas Matemáticas, 28 (2007): 99-108. 